\title{
The Tomato Kinome and the Tomato Kinase Library ORFeome: Novel Resources for the Study of Kinases and Signal Transduction in Tomato and Solanaceae Species
}

\author{
Dharmendra K. Singh, ${ }^{1}$ Mauricio Calviño, ${ }^{1}$ Elizabeth K. Brauer, ${ }^{1,2}$ Noe Fernandez-Pozo, ${ }^{1}$ Susan Strickler, ${ }^{1}$ \\ Roopa Yalamanchili, ${ }^{3}$ Hideyuki Suzuki, ${ }^{4}$ Koh Aoki, ${ }^{5}$ Daisuke Shibata, ${ }^{4}$ Johannes W. Stratmann, ${ }^{6}$ \\ George V. Popescu, ${ }^{1,7}$ Lukas A. Mueller, ${ }^{1}$ and Sorina C. Popescu ${ }^{1}$ \\ ${ }^{1}$ The Boyce Thompson Institute for Plant Research, Ithaca, NY 14853 U.S.A.; ${ }^{2}$ Department of Plant Pathology and Plant- \\ Microbe Biology, Cornell University, Ithaca, NY 14853 U.S.A.; ${ }^{3}$ Department of Plant Biology, North Carolina State University, \\ Raleigh, NC 27607, U.S.A.; ${ }^{4}$ Kazusa DNA Research Institute, Kazusa-Kamatari 2-6-7, Kisarazu 292-0818, Japan; ${ }^{5}$ Osaka \\ Prefecture University, 1-1 Gakuen-cho, Naka-ku, Sakai 599-8531, Japan; ${ }^{6}$ Department of Biological Sciences, University \\ of South Carolina, Columbia, SC 29208, U.S.A.; ${ }^{7}$ National Institute for Laser, Plasma \& Radiation Physics, Str. Atomistilor, \\ Nr. 409, Magurele 077125, Bucharest, Romania
}

Submitted 13 June 2013. Accepted 10 September 2013.

Protein kinase-driven phosphorylation constitutes the core of cellular signaling. Kinase components of signal transduction pathways are often targeted for inactivation by pathogens. The study of kinases and immune signal transduction in the model crop tomato (Solanum lycopersicum) would benefit from the availability of community-wide resources for large scale and systems-level experimentation. Here, we defined the tomato kinome and performed a comprehensive comparative analysis of the tomato kinome and 15 other plant species. We constructed a tomato kinase library (TOKN 1.0) of over 300 full-length open reading frames (ORF) cloned into a recombination-based vector. We developed a high-throughput pipeline to isolate and transform tomato protoplasts. A subset of the TOKN 1.0 library kinases were expressed in planta, were purified, and were used to generate a functional tomato protein microarray. All resources created were utilized to test known and novel associations between tomato kinases and Pseudomonas syringae $\mathrm{DC3000}$ effectors in a large-scale format. Bsk7 was identified as a component of the plant immune response and a candidate effector target. These resources will enable comprehensive investigations of signaling pathways and host-pathogen interactions in tomato and other Solanaceae spp.

An essential characteristic of organisms is the ability to sense and react to variations in the surrounding environment by activation of signal transduction networks. Protein kinases assemble in

\section{E. K. Brauer and N. Fernandez-Pozo contributed equally as third authors.}

TOKN 1.0 clones are available upon request from the Solgenomics website.

Corresponding author: Sorina C. Popescu; Telephone: +1.607.255.6578; E-mail: scp78@cornell.edu

* The $\boldsymbol{e}$-Xtra logo stands for "electronic extra" and indicates that five supplementary tables, three supplementary figures, and supplementary materials and methods information are published online.

(C) 2014 The American Phytopathological Society complexes and form pathways for signal sensing, intracellular transduction, and communication between cells (Wang et al. 2007). In plants, kinases have increased in number and diversity compared with other eukaryotes, reflecting the importance of plant kinases in growth, defense against pests, and adaptation to changing conditions (Lehti-Shiu et al. 2009).

Signal transduction pathways and protein kinases are at the core of the immune system. Signal transduction pathways activated during the plant immune response are composed of kinases belonging to diverse subfamilies and located in multiple cellular compartments. The activated kinases change the activation state or localization of their substrates and, thus, lead to changes in the expression of defense-related genes and metabolic pathways (Boller and Felix 2009; Zhang and Zhou 2010). Plasma membrane-associated kinase receptors (RLK, receptor-like kinases) mediate detection of pathogen-associated molecular patterns (PAMPs) and activate PAMP-triggered immunity (PTI) via mitogen-activated protein kinases and calcium-dependent protein kinases (Jones and Dangl 2006; Meng and Zhang 2013). Representatives of the receptor-like cytosolic kinases were shown to associate with active RLK complexes and to contribute to signal transduction innate immunity ( $\mathrm{Lu}$ et al. 2010; Shi et al. 2013). Both RLK and diverse cytosolic kinases are also involved in pathogen recognition and signal transduction during the effector-triggered immunity (ETI) (Day and He 2010; Hou et al. 2011; Tang et al. 1996).

The critical roles of kinases in establishing the plant immune response make them preferred targets for inactivation by pathogens. Bacterial effectors are macromolecules, which are secreted by bacteria, often directly into the infected host cells, in which they interact and inhibit the activity of kinases to undermine the immune response. Pathogen effectors are able to interfere with immune signaling by interacting with components in both PTI and ETI (Dou and Zhou 2012) and, thus, are under intense scientific scrutiny. The ability to identify, study, and perturb interactions established between pathogens and host molecules is essential to a better understanding of the pathogens and their hosts.

Tomato (Solanum lycopersicum) is an economically valuable crop and a widely used vegetable crop experimental model 
(Mueller et al. 2005; Van der Hoeven et al. 2002). Understanding kinase mechanisms in tomato is critical for basic research and has wider implications, as it will provide a key to understanding signal transduction networks in Solanaceae spp. However, the systematic study of tomato kinases is limited currently by the lack of a solid base of appropriate tools and reagents. Collections of individual ORF (open-reading frames) have been generated for several model organisms and have enabled systematic investigations of protein interactions (Arabidopsis Interactome Mapping Consortium 2011; Popescu et al. 2007a and b, 2009a and b; Reboul et al. 2003; Rual et al. 2004; Rubin et al. 2000). ORF libraries have also proved valuable for the experimental validation of bioinformatics-based gene and protein annotations of sequenced genomes. Cataloguing the full complement of tomato kinases (kinome) and construction of flexible ORFeome libraries are prerequisites for comprehensive investigations of kinases' enzymatic properties, interactomes, phosphorylation substrates, and biological functions.

Here, we report a set of novel resources and tools that enable systems biology investigations of signaling pathways and hostpathogen interactions in tomato. We defined the set of genes that constitutes the tomato kinome and compared these genes with their orthologs in other plants to find conserved and lineage-specific kinases. We constructed a tomato kinase library (TOKN 1.0) containing over 300 ORF cloned into a recombination-based vector. To demonstrate the utility of these resources, we utilized them to identify interactions between tomato kinases and Pseudomonas syringae effectors on tomato protein microarrays and in a split-luciferase complementation (SLC) screen in tomato cells.

\section{RESULTS}

\section{The tomato kinome.}

The tomato kinome was built by identifying genes encoding protein kinases $(\mathrm{PK})$ in the published tomato (Solanum lycopersicum) genome, using a hidden Markov model (HMM) analysis (Finn et al. 2011) over the Pfam-A database (Finn et al., 2010). We retrieved 1,436 putative kinase genes containing a kinase domain (Table 1). All identified genes, along with their best Pfam PK domain match and additional information, are listed in Supplementary Table 1. The annotated tomato genome contains 1,792 genes annotated as kinases (The Tomato Genome Consortium 2012). However, our analysis indicated that $356(20 \%)$ of these genes lack canonical eukaryotic protein kinase (ePK) domains, PK family signatures, or other kinase domains, suggesting that these genes may be currently misannotated, contain PK domains with low sequence similarity to domains in the Pfam-A, or have similarities with known kinases outside the PK domains.

For further insight into the TOKN 1.0, we classified the predicted tomato kinases in the COG (clusters of orthologous groups) database. Full-length predicted tomato protein se- quences were clustered with kinases from a group of 15 other species, representing a taxonomically diverse set with fully sequenced genomes. The group included eight dicots (Arabidopsis thaliana, Carica papaya, Cucumis sativus, Glycine max, Medicago truncatula, Populus trichocarpa, S. tuberosum, and Vitis vinifera), four monocots (Zea mays, Oryza sativa, Sorghum bicolor, and Brachypodium distachyon), one non-seed vascular plant (Selaginella moellendorffii), and two non-vascular plants (Chlamydomonas reinhardtii and Physcomitrella patens) (Fig. 1A). The COG analysis generated 2,040 clusters, out of which 661 contain tomato kinases. Of the total 1,436 tomato kinases with confirmed PK domains, 1,290 clustered in COG and 146 were singletons. An analysis of the COG database revealed that most of the tomato kinases are present as orthologous pairs with kinases from other plants. Moreover, while the majority of the tomato kinome shows conservation with vascular plant kinomes, several tomato kinases exhibit structural similarities with ancestral kinases from mosses, green algae, and ferns. Interestingly, 74 tomato kinases clustered only in Solanaceae-specific COG, while 170 tomato kinases clustered only in tomato-specific
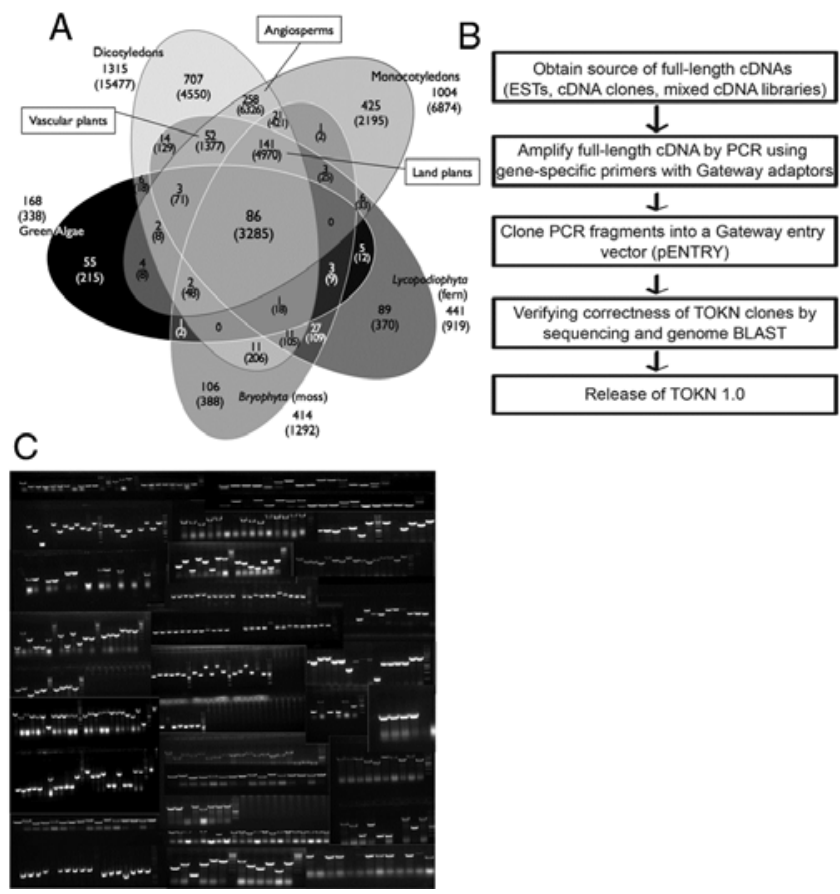

Fig. 1. Analysis of the tomato kinome and TOKN 1.0 library. A, Venn diagram comparison of the Cluster of Ortologous Groups (COG) database containing kinases from 16 plant species. The number of genes in each COG is shown in parentheses, while the numbers of COG and genes in each COG are shown outside the diagram. B, Schematic representation of the pipeline for developing TOKN 1.0. C, A composite image of the gel electrophoresis assays of the polymerase chain reaction products, representing the tomato kinase cDNAs subcloned into an entry Gateway-compatible vector.

Table 1. Summary of the tomato kinome and a tomato kinase library (TOKN v1.0) collection

\begin{tabular}{|c|c|c|c|c|}
\hline \multirow[b]{2}{*}{ Parameter } & \multicolumn{2}{|c|}{ Tomato kinome } & \multicolumn{2}{|c|}{ TOKN1.0 } \\
\hline & \# & $\%$ & $\#$ & $\%$ \\
\hline Kinase genes & 1,436 & 100 & 285 & $19.85^{\mathrm{a}}$ \\
\hline Classified in clusters of orthologous groups (COG) database & 1,290 & 89.83 & 277 & 97.19 \\
\hline Singletons & 146 & 10.17 & 8 & 2.81 \\
\hline Annotated as kinase (ITAG v2.3) & 1,365 & 95.06 & 285 & 100.00 \\
\hline Supported by a Pfam domain & 1,436 & 100.00 & 285 & 100.00 \\
\hline COG numbers & 661 & & 170 & $25.72^{\mathrm{a}}$ \\
\hline Putative tomato-specific kinases & 170 & 11.82 & 9 & 3.16 \\
\hline Putative Solanaceae-specific kinases & 74 & 5.15 & 4 & 1.40 \\
\hline
\end{tabular}

\footnotetext{
a Percentage based on the tomato kinome as $100 \%$.
} 
COG. These 244 kinases (also including the 146 singletons) appear to have a higher sequence divergence from the other plant kinases, as compared with the rest of the tomato kinases, and thus, they may constitute tomato- or Solanum-specific kinases. We found that $54 \%$ of these kinases have sequence similarity to RLK. Notably, the leucine-rich repeat (LRR)-RLK have a high representation within this set (Supplementary Table 2), reinforcing the previous observation that LRR-RLK underwent a significant expansion in tomato relative to other plants (Sakamoto et al. 2012).

To evaluate the potential of tomato as a model crop system for the study of kinases and signal transduction, we compared the tomato kinome with Arabidopsis, a well-annotated model plant. A majority of predicted tomato kinase gene clustered in COG with Arabidopsis kinases. Tomato and Arabidopsis have similar numbers of kinases in $85.5 \%$ of the common COG and the percentages of kinase-encoding genes in the tomato and Arabidopsis genomes are comparable $4.14 \%$ (1,436 PK out of 34,727 genes) in tomato versus $4.93 \%$ (1,352 PK out of 27,416 genes) in Arabidopsis. Taken together, these analyses indicate that tomato is an excellent model for kinome studies in Solanaceae spp.

\section{Construction of TOKN 1.0, a full-length tomato kinase ORF library.}

To facilitate large-scale studies of the tomato kinome, we generated TOKN 1.0, a collection of full-length tomato kinase ORF representing $20 \%$ of the kinome and $25 \%$ of its PK. To generate TOKN 1.0, we selected kinases for which full-length cDNA or complete or partial expressed sequence tag (EST) sequence information was available. The majority of the kinases included in TOKN $1.0(97 \%)$ clustered with orthologous kinases from other plant species in 170 distinct kinase COG. Nine kinases in TOKN 1.0 are specific for tomato, while four are found only in the tomato and potato kinomes (Table 1) and may represent lineage-specific kinases.

To build TOKN 1.0, full-length tomato kinase ORF were amplified from various sources of genetic material. The polymerase chain reaction (PCR) fragments were transferred into the pENTR Gateway vector to generate TOKN 1.0 (Fig. 1B). The tomato ORF in TOKN are, on average, $1.5 \mathrm{~kb}$, with a maximum length of $3.2 \mathrm{~kb}$ (Fig. 1C). The ORF are in an "open" configuration - they lack a stop codon to enable C-terminal tag attachment. A Gene Ontology (GO) analysis of the Arabidopsis orthologs of ORF revealed significantly enriched GO categories in TOKN 1.0, including "protein metabolism" (12.5\%), "response to stress" (10\%), and "plasma membrane" (36\%). Furthermore, approximately $22 \%$ of the TOKN kinases were predicted to contain single or multiple putative transmembrane domains (Supplementary Table 3).

The quality of all TOKN clones was evaluated by sequencing from both $5^{\prime}$ and $3^{\prime}$ ends, followed by alignment with the tomato genome to verify the identity of the ORF and the gene exonintron structure. Of the 389 attempted, we successfully cloned and validated 306 ORF (78.7\%). The majority $(96.08 \%)$ of the cloned ORF had the expected length and correct exon-intron structure, as shown by genome alignment. Notably, in the case of the failed ORF, experimental validation for the gene exonintron structure is not yet available. In these cases, the primers for PCR amplification were designed based on the predicted gene structures and failed to produce valid PCR products. These results suggest that the current tomato genome may contain annotations with erroneously calculated exon-intron structures for which experimental verification is required.

Altogether, TOKN 1.0 comprises 306 sequence-verified tomato ORF, representing a resource of 285 unique full-length kinases cloned into a Gateway-compatible vector. We envision that TOKN 1.0 will facilitate large-scale protein functional studies in tomato and in other Solanaceae spp. for which ORF collections are not yet available.

\section{A method for the extraction and transformation of tomato protoplasts for large-scale applications.}

Protoplasts are a suitable system for studying proteins in a native context (Faraco et al. 2011; Walter et al. 2004). However,
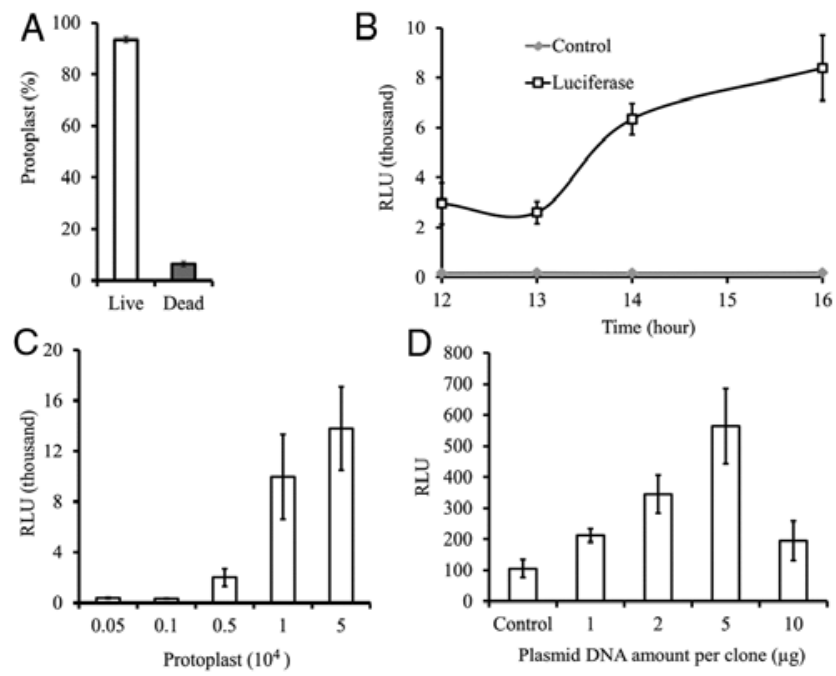

Fig. 2. Optimization of tomato protoplast extraction and transformation parameters. A, Qualitative analysis of the tomato protoplasts. Tomato leaves were harvested and digested for 12 to $13 \mathrm{~h}$ to release the mesophyll protoplasts. The extracted protoplasts were stained with Evan's blue to reveal dead protoplasts. The values represent the mean of three biological replicates \pm standard deviation (SD). B, Optimization of the posttransformation incubation time. Protoplasts were transformed with $0.5 \mu \mathrm{g}$ of full-length luciferase, and the luminescence was measured at the indicated time points. The values represent the mean of four independent technical replicates \pm SD. Two more biological replicates gave similar results. C, Optimization of the protoplast amount. The indicated numbers of protoplasts were transformed with $0.5 \mu \mathrm{g}$ of full-length luciferase. The values represent the mean of four independent technical replicates \pm SD. D, Optimization of the amount of plasmid DNA for protoplast cotransformation. Protoplasts were cotransformed with Pto-NLuc and AvrPto-CLuc. The values represent the mean of four independent technical replicates \pm SD. Two more biological replicates gave similar results.

Table 2. Parameters and throughput in protoplast preparation and transformation methods

\begin{tabular}{|c|c|c|c|c|}
\hline Plant species & Number $^{\mathrm{a}}$ & Plasmid amount for protoplast transformation & Throughput & Reference \\
\hline Tomato & $1 \times 10^{4}$ & $5 \mu \mathrm{g}$ each construct (RLucN and RLucC) & Up to six 96 -well plates at one time & This publication \\
\hline Tomato & $3.5 \times 10^{4}$ & $40 \mu \mathrm{g}$ & N/A & Devarenne et al. 2006 \\
\hline Tomato & $3 \times 10^{5}$ & $5 \mu \mathrm{g}$ per two constructs (CoIP) & Four samples & Hahn et al. 2011 \\
\hline Arabidopsis & $2 \times 10^{4}$ & $10 \mu \mathrm{g}$ per construct (FLucN and FLucC) & Maximum 20 transfections at one time & Li et al. 2011 \\
\hline Arabidopsis & $2 \times 10^{5}$ & N/A & 24-well microtiter plates & Chen et al. 2008 \\
\hline Arabidopsis & $5 \times 10^{3}$ & $10 \mu \mathrm{g}$ per construct (RLucN and RLucC) & 96-well plates & Fujikawa 2007 \\
\hline Rice & $2 \times 10^{5}$ & $10 \mu \mathrm{g}$ for $\mathrm{BiFC}, 15 \mu \mathrm{g}$ for $\mathrm{Co}-\mathrm{IP}$ & Low throughput & Zhang et al. 2011 \\
\hline
\end{tabular}

a Protoplasts per transformation. 
current methods are not amenable for large-scale experimentation, due to factors such as inefficient protoplast extraction and the requirement of large amounts of plasmid DNA (plDNA) for transformation (Devarenne et al. 2005; Horvath 2009; Nguyen et al. 2010) (Table 2). To develop a high throughput-amenable method, we identified the optimal parameters for extracting abundant high-quality protoplasts. We determined that the leaves from the third- to fifth position of approximately 4-week-old plants, grown under natural light and devoid of any visible signs of stress, yielded a large number $(>94 \%)$ of viable protoplasts (Fig. 2A; Supplementary Fig. S1a to c). Next, we focused on optimizing two critical parameters for protoplast transformation: the protoplast incubation time after transformation with plDNA and the number of protoplasts per well in a 96-well plate. To optimize the incubation time, $40 \mu \mathrm{l}$ of $5 \times 10^{6} / \mathrm{ml}$ protoplasts were incubated in the dark for 12 to $16 \mathrm{~h}$ after transformation with 0.5 $\mu \mathrm{g}$ of Renilla reniformes luciferase, followed by measurement of the luciferase activity at multiple time points after transformation. We found that the post-transformation incubation time greatly affected protoplast transformation efficiency. The luminescence intensity was similar at 12 and $13 \mathrm{~h}$ and increased 2.5fold at $14 \mathrm{~h}$ and by only about 1.3 -fold at $16 \mathrm{~h}$, relative to the background luminescence measured in controls (Fig. 2B). Similarly, to determine the optimal number of protoplasts per well in a 96-well plate, we transformed a fixed amount of full-length Renilla reniformes luciferase into a range of protoplast concentrations and measured the luciferase activity. We found that the activity was significantly higher when using $1 \times 10^{4}$ to $5 \times 10^{4}$ protoplasts compared with either higher or lower amounts (Fig. $2 \mathrm{C}$ ). We determined that highly viable tomato protoplasts could be obtained in quantities sufficient for ten to twelve 96-well plates $\left(50 \times 10^{6}\right.$ to $60 \times 10^{6}$ protoplasts $)$ in one experiment from 32 approximately 1 -month-old plants.

Altogether, we demonstrated that our protoplast purification and transformation protocol is more efficient than the previously reported assays. A detailed protocol is presented in the Supplementary Materials and Methods.

\section{A standardized tomato split-luciferase complementation method for screening protein interactions.}

To our knowledge, a high-throughput system for detecting in vivo protein-protein interactions (PPI) is not yet available for tomato. We set out to develop a standardized large scaleamenable tomato SLC method using a set of recombinationbased vectors (Fujikawa 2007). To benchmark the sensitivity of the tomato SLC, we used the well-studied tomato cytosolic kinase Pto and the P. syringae effector AvrPto. Specifically, we tested two reference interaction sets (RIS), the AvrPto-Pto (Bogdanove and Martin 2000; Tang et al. 1996) as a positive reference set (PRS), and the AvrPto ${ }^{\text {I96A }}$-Pto (Nguyen et al. 2010) as a negative reference set (NRS). For the SLC, protoplasts were extracted from the leaves of tomato variety Rio Grande prf3 to prevent AvrPto-triggered cell death (Nguyen et al. 2010). Protoplasts were co-transformed with $5 \mu \mathrm{g}$ each of the kinase and effector plasmids, as we found that this amount of pIDNAs produced an activity significantly higher than either

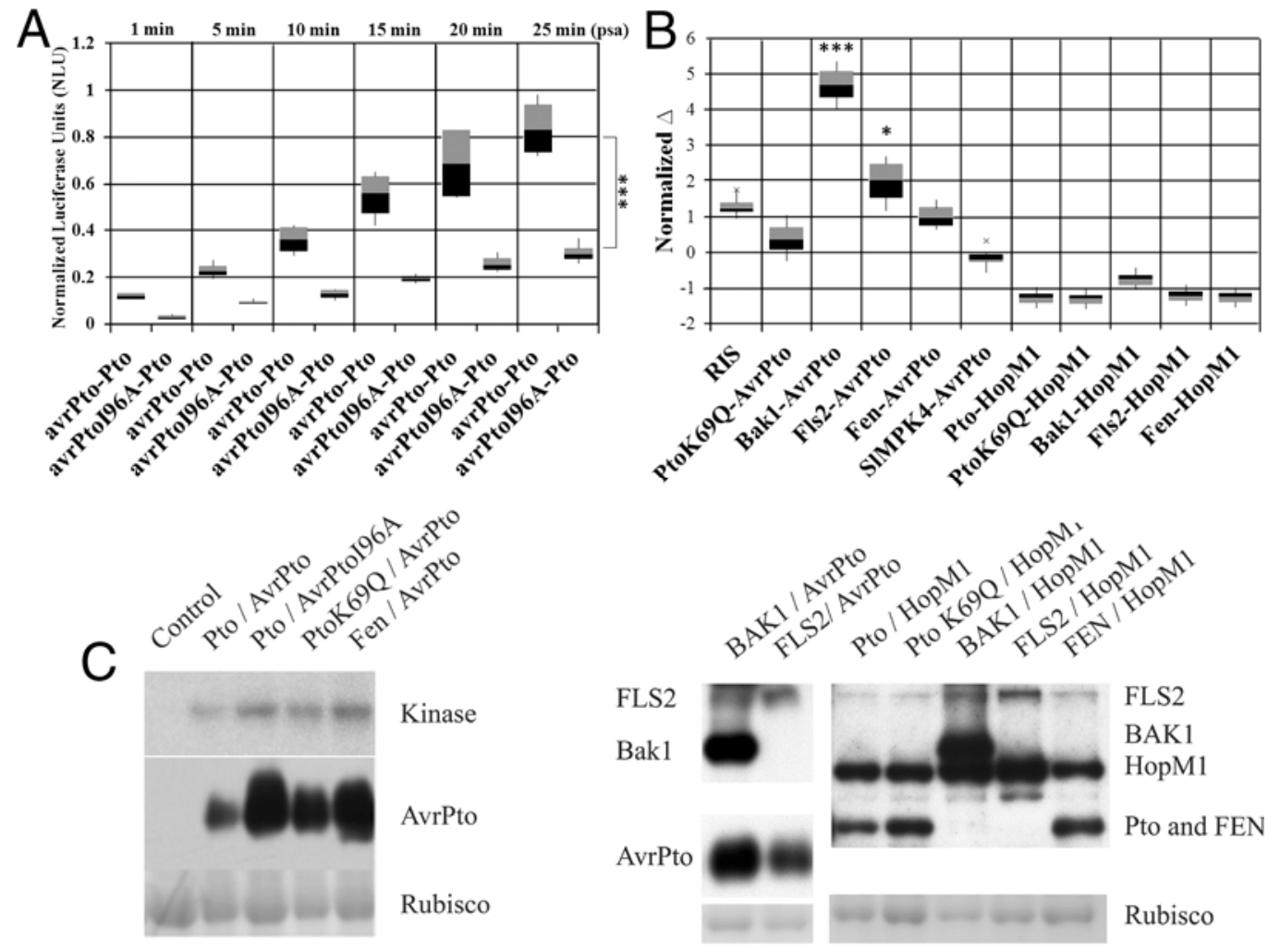

Fig. 3. Standardizing the tomato luciferase complementation method using a reference set of protein-protein interactions. A, Time-dependent dynamics of the interaction between the tomato kinase Pto and the Peudomonas syringae effector AvrPto (positive reference set [PRS]) or the defective AvrPto ${ }^{\text {I96A }}$ (negative reference set [NRS]). The box plot represents normalized luminescence units for each interaction at six time points, 5-min intervals, postsubstrate addition (psa), from three biological replicates, each with four technical replicates per interaction set. The Euclidean normalized distance ( $\Delta$ ) between PRS and NRS sets equals an approximately threefold normalized luciferase units difference. B, Detection of kinase-effector interactions by split-luciferase complementation. The box plot represents the $\Delta$ for the reference interaction sets (RIS) and the tested interactions. Statistical significance of the difference between the normalized mean intensity of candidate and reference datasets was calculated using the $Z$ test. The $*$ symbols represent levels of significance ( $*: Z>$ e- 03 , $*: Z>\mathrm{e}-06$; ***: $Z>\mathrm{e}-09$ ). The data is representative of two biological replicates, with four technical replicates per each interaction set tested, that gave similar results. C, Representative immunoblots of protein sets coexpressed in the protoplasts used in (A and B). Proteins were visualized using antibodies against the $\mathrm{N}$ - or $\mathrm{C}$-half luciferase fragments. Ponceau staining shows Rubisco as a loading control. 
lower or higher amounts (Fig. 2D). We considered the $5 \mu \mathrm{g}$ per construct plDNA amount optimal for our assay and indicative of the performance of other kinase-effector pairs. Following cotransformation, the activity of the reconstituted luciferase was measured over time to obtain a dynamic view of the interactions. The PRS-transformed protoplasts showed significantly higher levels of activity relative to the NRS-transformed protoplasts (Fig. 3A). The difference in activity between the two RIS was threefold, indicating that this threshold is a practical cutoff for calling positive SLC interactions.

To further test the threefold threshold for identifying SLC interactions, we expanded our kinase-effector list with other known positive or negative interactions of AvrPto. We included the tomato Fen, the variant Pto ${ }^{\mathrm{K} 69 \mathrm{Q}}$ (Scofield et al. 1996; Tang et al. 1996), and SIMPK4 as negative interactors, and the Arabidopsis AtFLS2 and AtBAK1 as a positive and unresolved interactor, respectively (Shan et al. 2008; Xiang et al. 2011). The HopM1 effector, which has never been shown to interact with kinases (Nomura et al. 2006), was included as a control. Following normalization, statistical testing using the RIS standards was used to select the PPI from SLC measurements. We found that AvrPto-AtFLS2 and AvrPto-AtBAK1 (or the tomato ortholog-SIBAK1, not shown) tested positive, while all the other AvrPto- or HopM1-containing sets tested negative (Fig. 3B). Extracts from cotransformed protoplast showed that recombinant proteins were expressed at comparable levels (Fig. 3C).

Our results indicate that the tomato SLC has high accuracy and sensitivity in identifying PPI and demonstrate the performance of the method in a large-scale experimental setting.

\section{The tomato split-luciferase complementation method identifies novel effector targets in tomato.}

To explore the value of TOKN 1.0 as a resource for investigating host-pathogen interactomes and further test the standardized tomato SLC for detecting PPI, we performed a targeted tomato SLC screen with a set of tomato kinases and $P$. syringae effectors. We included in the screen the tomato orthologs of known Arabidopsis kinases targeted by P. syringae effectors and the respective effectors (Table 3; Supplementary Fig. 2). We found that HopAI1 interacted with both SIMPK4 and SIMPK6, while it weakly interacted with another kinase, SIMKK2 (Fig. 4A). Notably, SIMPK6-HopF2 interacted with a higher affinity than SIMPK4-MPK6. Both AtMPK6 and AtMPK4 were previously shown to interact with HopAI1 (Zhang et al. 2012). Another effector tested, HopF2, was previously shown to bind AtMKK5 (Wang et al. 2010).

We found that HopF2 strongly interacted with SIMKK5, while the related SIMKK2 showed a much weaker binding to HopF2 (Fig. 4B). Interestingly, HopF2 also interacted with SIMPK6 in tomato SLC. In a previous study, although AtMPK6 failed to coimmunoprecipitate with $\mathrm{HopF} 2$, its kinase activity was reduced following incubation with HopF2 (Wang et al. 2010). None of the tested tomato kinases interacted with HopM1 in tomato SLC (Fig. 4C).

Next, we found that one of the TOKN kinases, Solyc11g064890.1 (SlBSK7), interacted with HopAI1 and
HopF2 but not HopM1 (Fig. 4A to C). SlBsk7 is a predicted cytosolic RLK orthologous to AtBsk7 (Fig. 4D). The Arabidopsis brassinosteroid signaling kinases (Bsk) genes constitute a large gene family with members critical for brassinosteroid (BR) signal transduction and plant immunity (Shi et al. 2013; Tang et al. 2008). To test a possible immune function of SlBsk7, we used viral-induced gene silencing (VIGS) and the cell death (CD)-protection assay, in Nicotiana benthamiana (Chakravarthy et al. 2009; Oh and Collmer 2005). Leaves from plants silenced for $B s k 7$ or $F l s 2$ were infiltrated with the nonpathogenic PTI-inducing Pseudomonas fluorescens and at $7 \mathrm{~h}$, overlapping regions infiltrated with the ETI-inducing strain $P$. syringae pv. tomato DC3000. The intensity of CD in the overlapping regions was scored at 6 days after infiltrations (Fig. 4E). In accordance with Chakravarthy and associates (2009), PTI induced by $P$. fluorescens inhibits the appearance of CD in the $P$. fluorescens $-P$. syringae pv. tomato DC3000 intersecting area; on the other hand, in plants with impaired PTI signaling, $\mathrm{CD}$ is triggered and visible in the intersecting area. We found that $N$. benthamiana plants silenced for $N b B s k 7$ exhibited significantly higher $\mathrm{CD}$ in the $P$. fluorescens $-P$. syringae pv. tomato DC3000 overlapping areas compared with the negative controls. Overall, the percentage of areas showing $\mathrm{CD}$ in $N b B s k 7$-silenced plants was similar to the percentage measured in the positive control, the $N b F l s 2$-silenced plants. None of the infiltrated constructs induced $\mathrm{CD}$ or other phenotypes outside the infiltrated areas. We confirmed that the abundance of $B s k 7$ mRNA in the silenced plants was up to threefold lower than in the controls (Fig. 4F). Our results suggest that Bsk7 might be a component of the PTI or ETI-related hypersensitive response in $N$. benthamiana. The interactions between SIBSK7 and HopAI1 or HopF2 remain to be confirmed and their functional significance tested in vivo.

In summary, we observed a good correlation between the PPI decisions from the tomato SLC and the PPI reported in the literature. Thus, we demonstrated that, using the standardized SLC, we confirmed known and discovered new candidate immune kinase targets.

\section{Production of TOKN 1.0 protein microarrays \\ for high-throughput detection of kinase-protein interactions.}

Purified recombinant proteins can be used to generate protein microarrays (PMA) for the large-scale study of protein interactions and enzymatic properties (Popescu and Popescu 2011; Popescu et al. 2007b, 2009a). To test the utility of TOKN 1.0 clones as a resource to study protein function, we generated a tomato PMA comprised of 59 tomato kinases (Supplementary Table $4 \mathrm{a}, \mathrm{b}$ ) selected from a group of TOKN kinases with potential roles in the immune response, based on homology to Arabidopsis kinases and preliminary screening of TOKN (data not presented). In addition, five Arabidopsis kinases with known or putative roles in plant immunity, including FLS2 and BAK1, were added to the PMA as controls. To produce the PMA, we generated an expression library by transferring 59 of TOKN 1.0 gene inserts into a plant binary transformation vector. The clones were expressed in N. ben-

Table 3. Tomato kinase orthologs of known kinase targets of Pseudomonas syringae effectors, tested using tomato split luciferase assay (SLC)

\begin{tabular}{|c|c|c|c|c|c|}
\hline Effector & Kinase & TomatoSLC & For Arabidopsis & Reported method of interaction $^{a}$ & Reference \\
\hline AvrPto & SIBAK1 (Solyc10g047140) & Positive & Positive/negative & $\begin{array}{l}\text { Co-IP in Arabidopsis protoplasts and yeast split- } \\
\text { ubiquitin/Co-IP and BiFC in Arabidopsis protoplasts }\end{array}$ & $\begin{array}{l}\text { Shan et al. 2008; } \\
\text { Xiang et al. } 2011\end{array}$ \\
\hline HopAI1 & SIMPK4 (Solyc01g094960) & Positive & Positive & Co-IP in planta & Zhang et al. 2012 \\
\hline HopAI1 & SIMPK6 (Solyc12g019460) & Positive & Positive & GST pull-down in Escherichia coli, Co-IP in planta & Zhang et al. 2007 \\
\hline HopF2 & SIMPK6 (Solyc12g019460) & Positive & Negative & GST pull-down in $E$. coli & Wang et al. 2010 \\
\hline HopF2 & SIMKK5 (Solyc03g123800) & Positive & Positive & GST pull-down in E. coli & Wang et al. 2010 \\
\hline
\end{tabular}

${ }^{\mathrm{a}} \mathrm{Co}-\mathrm{IP}=$ co-immunoprecipitation, $\mathrm{BiFC}=$ bimolecular fluorescence complementation, GST = glutathione-S-transferase. 

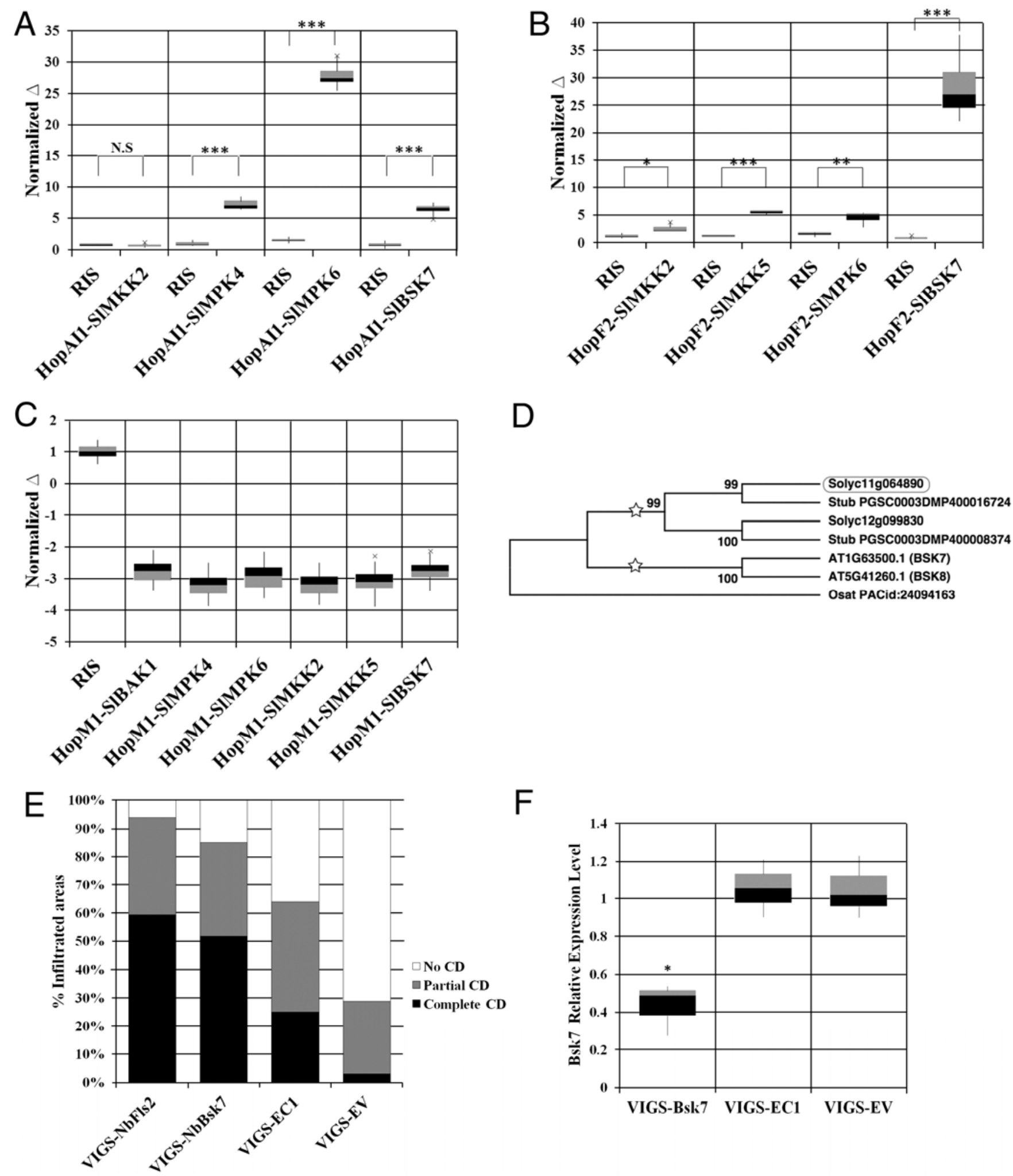

Fig. 4. The tomato split-luciferase complementation (SLC) assay identifies tomato kinases that interact with Pseudomonas syringae effectors. A targeted SLC screen to identify A, HopAI1- and B, HopF2-, and C, HopM1-interacting kinases. The box plots shows the normalized distances $(\Delta)$ for the reference interaction sets (RIS) and tested interactions. The interactions were measured in biological replicates in 96-well plates, each with their own set of RIS. RIS were used for normalization and for statistical testing $(Z$ test). The * symbols indicate levels of significance (**: $Z>\mathrm{e}-06$; ***: $Z>\mathrm{e}-09)$. D, Cladogram of BSK7 orthologs selected from the clusters of orthologous groups (COG) gf_000275, including genes from tomato (Solyc, circled), potato (Stub), Arabidopsis, and a monocot (rice, Osat) as an outgroup. Stars indicate putative duplication events and numbers represent bootstrapping values. E, Cell death (CD)-protection assays in Nicotiana benthamiana silenced for NbBSK7, NbFLS2, or the negative controls, the empty vector TRV2 (EV) or EC1. The bars represent percentage of CD in P. florescence-P. syringae DC3000 intersecting areas from three biological replicates (each with 7 to 9 plants per construct tested) and show $\mathrm{CD}$ intensity ranging from complete $\mathrm{CD}$ (black) to no $\mathrm{CD}$ (white). The $\mathrm{CD}$ in the overlapping region in the NbBSK7-silenced plants was significantly different compared with EV $(P$ value $=7.09 \mathrm{e}-07)$ and EC1 $(P$ value $=2.99 \mathrm{e}-03)$ calculated using Student's $t$-test. F, Quantification of $B s k 7$ transcript accumulation in $N$. benthamiana silenced with $N b B S K 7$ or the controls EV or EC1. The box plots represent data from three replicates for EC1 and $N b B s k 7$-silenced plants and two replicates for EV. * shows statistical significance $(P<0.05)$ using Student's $t$-test. 
thamiana (Supplementary Fig. 3a) and the recombinant kinases were purified. To analyze the activity of the purified kinases, 26 were tested in an in vitro kinase assay, with conditions optimized to detect a wide range of active kinases. Seven of the tested tomato kinases demonstrated autophosphorylation at the correct band size for the full-length protein, indicating that they are active. The kinases not showing activity in these assays either are kinases with weak autophosphorylating activity (such as FLS2) or may require optimized conditions to show activity.

The entire set of 66 recombinant purified Arabidopsis and tomato proteins were used to generate PMA. The presence and relative abundance of the proteins on the PMA was verified by immunoassay (Fig. 5A). The PMA were used to identify PPI by probing them with either AvrPto-V5 or the AvrPto ${ }^{\text {I96A }}-\mathrm{V} 5$ fusion proteins. Kinase-effector complexes were detected by probing the slide with an anti-V5 Cy5-labeled antibody and quantifying the resulting fluorescence (Fig. 5B). To demonstrate how the assay performs with previously known interactors, we highlighted the interactions of AvrPto with AtFLS2 or AtBAK1 in Figure 5B and C. In line with previously published results, FLS2 demonstrated binding to the native AvrPto but not to the AvrPto ${ }^{\text {I96A }}$. In contrast, AtBAK1 did not bind AvrPto or $\mathrm{AvrPto}^{\mathrm{I}}{ }^{\mathrm{A}} \mathrm{A}$. Of the remaining 64 printed kinases, 26 were identified as novel putative interactors found for AvrPto and 22 as putative interactors of AvrPto ${ }^{196 \mathrm{~A}}$. Over $50 \%$ of the putative AvrPto interactors are RLK, including the SlBSK7 shown above to be important for PTI. Other putative AvrPto interactors include orthologs of Arabidopsis kinases known to be involved in brassinosteroid signaling and defense, including BSK8, NIK1, and RLK1. All putative interactors will require further validation. Our results indicate that TOKN 1.0 PMA contain functional proteins and are a resource for characterizing kinase function and interactions.

\section{DISCUSSION}

In this work, we described a set of resources and methods that establish a basis for functional high-throughput analyses of kinases and signal transduction pathways in tomato, a model system for Solanaceae spp. We generated i) a tomato kinome containing a high-confidence set of protein kinases, ii) the TOKN 1.0, a full-length ORF library of experimentally verified tomato kinases, easily transferable to other destination vectors, iii) a standardized method for the tomato protoplast extraction, transformation amenable for large scale PPI screens, and iv) a tomato PMA containing a subset of the TOKN 1.0 kinases. We demonstrated that these resources can be used to identify known and new candidate effector targets.

The tomato kinome described here contains 1,436 kinases with confirmed PK domains. Our predictions solidify current tomato kinase annotations and provide kinase classification and functional descriptions. In addition, our comparative study between tomato kinases and their orthologs in other plants provides information on the size and composition of diverse kinase subfamilies in plants, facilitates selection of orthologs across plant species, and transfers knowledge from well-studied model systems such as Arabidopsis to crop plants. The TOKN library contains $25 \%$ of the predicted tomato kinome. Notably, most of the TOKN kinase genes were found to be expressed in the leaf (Supplementary Table 5). We tested the activity of a subset of the TOKN 1.0 library kinases and found that $27 \%$ demonstrated autophosphorylation activity. A comparable number (24\%) of Arabidopsis kinases demonstrated autophosphorylation activity, using a wheat germ cell-free system (Nemoto et al. 2011) and on Arabidopsis protein microarrays (Popescu et al. 2009a). Both the tomato kinome and TOKN 1.0 library are sources of functional tomato kinases and starting points for building an interactomics resource base for family Solanaceae.

We developed a tomato SLC method amenable to largescale approaches and demonstrated that it detects interactions with high sensitivity and specificity between kinases and $P$. syringae effectors. Our method was benchmarked using a reference set of positive and negative kinase-effector sets in a highthroughput setting. Particularly, we identified new candidate targets of $P$. syringae effectors and showed that one of them, $B s k 7$, is a promising element of immune signaling pathways in $N$. benthamiana. The ability of multiple BSK proteins to establish direct interactions with RLK and cytosolic kinases is critical to their functions in BR-mediated plant growth and PAMPmediated signaling. Several Arabidopsis BSK genes, including $A t B S K 7$, were shown to interact and to be phosphorylated by both BRI1 and BIN2 (Sreeramulu et al. 2013; Tang et al. 2008), and AtBSK1 was found to bind FLS2 and modulate flg22-mediated signaling (Shi et al. 2013). We hypothesize that multiple BSK are able to interact with pathogen effectors and participate in immune signaling. Apart from BSK7, its closest homolog, BSK8, has 99\% identity with BSK7 and is a
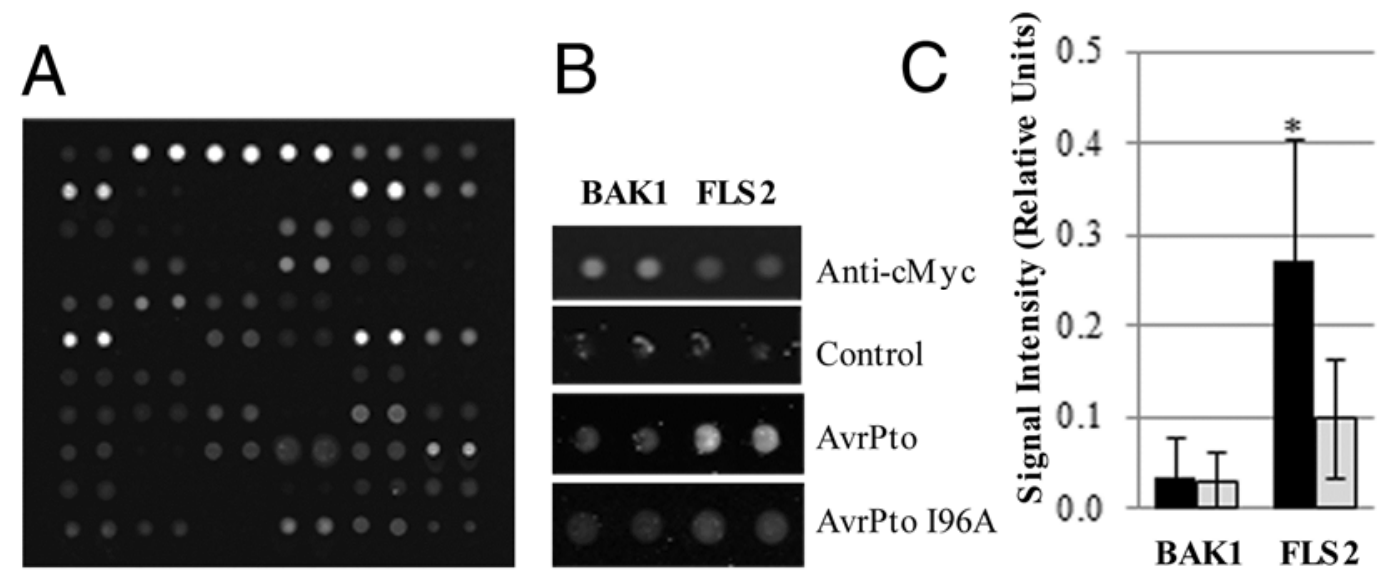

Fig. 5. Analysis of AvrPto effector-kinase interactions using the tomato kinase library (TOKN 1.0) protein microarray. A, Immunoassay of a protein microarray to detect the amount of each spotted kinase. Each purified kinase was spotted in duplicate and two slides were used per assay. The amount of protein is indicated by signal from black (lowest) to white (highest). B, Images showing BAK1 and FLS2 printed on protein microarrays used in an immunoassay (Anti-cMyc), control, AvrPto probing, and AvrPto ${ }^{196 \mathrm{~A}}$ probing assays. C, Results of quantification of the protein interaction after subtracting control signals and normalizing to immunoassay signals. Each bar represents an average of two replicate spots on two separate slides and the standard deviation of the signal. * indicates interaction as decided based on significant difference from a negative control kinase $(t$-test $\alpha<0.01)$. 
likely candidate. Notably, BAK1, another critical component of the BR-mediated and PTI signaling pathway, is also targeted by $P$. syringae effectors (Shan et al. 2008). It is possible that effectors preferentially target signaling elements critical for pathogen perception that have dual functions in growth and immunity, a hypothesis that remains to be further investigated.

We showed that tomato PMA could be produced and employed to detect interactions between kinases and effectors. Compared with SLC, PMA are a faster method for testing PPI. PMA can be used to screen thousands of potential PPI within days (Azmi et al. 2012; Popescu et al. 2007b), while it would take months to screen the same interactions using SLC. Although not shown here, PMA can be used to detect autophosphorylation of the printed kinases and changes in their phosphorylation activity following exposure to a probe of interest (Popescu et al. 2009a). PMA also offer a higher level of control over assay conditions compared with SLC, as well as the ability to assess the amount and integrity of the proteins used to measure the interaction (Kaushansky et al. 2010). However, unlike SLC, PMA detect direct PPI, outside of the context of the cell, and thus, several factors that may influence kinaseeffector interactions, such as compartmentalization and posttranslational modifications (Dou and Zhou 2012; Feng et al. 2012; Shan et al. 2000), are excluded. Thus, an advantage of the SLC method is that it detects interactions occurring in an endogenous cellular context in which specific protein modifications are maintained. In this context, when comparing the SLC with PMA results regarding AvrPto interaction with BAK1 or FLS2, an interesting picture emerges. The AvrPtoAtFLS2 interaction was identified as positive by both methods, while the disputed AvrPto-BAK1 interaction differed between the methods. Both AtBAK1 and SlBAK1 interacted with AvrPto in tomato SLC, while on PMA, AtBAK1 was unable to bind AvrPto. This discrepancy is not unprecedented, since conflicting results regarding BAK1-AvrPto interactions have been disputed in the literature (Xiang et al. 2011). Thus, it is important to understand the biases of any detection method when studying PPI. Many protein interactions in the cell occur in the context of multiple protein complexes and may be species-specific. Certain interactions may be detected in an in vivo system such as SLC but not in in vitro systems such as PMA. Thus, an optimal approach to determining PPI in a high-throughput manner would incorporate both in vivo and in vitro methods to capitalize on the advantages of each system.

We found that AvrPto interacted with approximately half of the proteins on the PMA, which is a higher proportion of interactions than has been measured in other PMA studies (Popescu et al. 2009b). While some of these interactions may not occur in vivo due to the reasons outlined above, this result supports the hypothesis that AvrPto is able to bind and potentially target many different kinases. This idea has been suggested previously and is based on the evidence that AvrPto is a potent inhibitor of PAMP-induced signaling, can overcome nonhost resistance, and binds multiple RLK (Martin and Kamoun 2012). The efficacy of AvrPto's suppression of early signaling is surprising, considering that plants detect many different PAMPs and there is significant redundancy within signal transduction cascades in plants. Together with our findings, this suggests AvrPto may have evolved to bind multiple kinases to interrupt pathogen-induced signaling and prevent PTI. Thus, by measuring PPI both in vivo and in vitro, we identified putative effector interaction partners that provide insights into AvrPto functions.

In conjunction to other resources and the vast amount of genomic tools available for tomato, the resources we described here offer opportunities for functional large-scale analyses. The applicability of these resources is very broad, and we envision that it will facilitate functional studies in other Solanaceae spp. for which ORF collections are not available.

\section{MATERIALS AND METHODS}

\section{Bioinformatics for kinase analyses.}

To generate the tomato kinome, all sequences from ITAG 2.3 were analyzed with the command line version of HMMER v3.0 (Finn et al. 2011) with Pfam-A as HMM database (Finn et al. 2010). Genes with an ePK domain (e value $<1$ e-5) were selected. The Arabidopsis kinome was obtained using a TAIR (The Arabidopsis Information Resource) search with the GO0016301 term "kinase activity." Kinase COG were obtained using OrthoMCL software v2.0.3 (Li et al. 2003) and default parameters $(\mathrm{e}$ value $=1 \mathrm{e}-5$ and inflation value $=1.5)$. Blast v2.2.26+ (Altschul et al. 1997) was used for the "all versus all" protein comparison step. Fasta files of protein sequences (without splice variants) and the annotations for all species, except tomato and Arabidopsis, were downloaded from Phytozome v. 9.0 (Goodstein et al. 2012). Data for tomato and Arabidopsis were downloaded from the Sol Genomics Network (SGN) (Bombarely et al. 2011) and TAIR10 (Garcia-Hernandez et al. 2002), respectively. SGN servers running Ubuntu Linux, with 64 cores and 512 or 1 TB of RAM memory, respectively, were used for the analysis. Protein transmembrane domains were predicted with TMHMM v2.0 (Moller et al. 2001). To calculate gene expression values, tomato RNA-Seq reads from leaf RNA were obtained from GenBank SRA study SRP010775, were cleaned with fastq-mcf (ea-utils FASTQ processing utilities website), and were mapped to the $S$. lycopersicum 'Heinz' SL2.40 genome using Tophat2 (Kim et al. 2013). Cufflinks (Trapnell et al. 2012) was used to calculate FPKM (fragments per kilobase of transcript per million mapped reads) values. Phylogenetic trees were obtained using MEGA 5 (Tamura, et al. 2011), based on multiple full-length protein alignment using MUSCLE (Edgar 2004) and the maximum likelihood with 1,000 Bootstrap replications.

\section{Construction of the tomato ORF kinase library.}

Predicted kinase coding sequences based on ITAG2.3 S. $l y$ copersicum 'Heinz 1706' were screened against the S. lycopersicum EST databases using BLASTn to find full-length (100\% match) EST and EST containing start codons. Start codoncontaining EST were identified by matches that contained part of the $5^{\prime}$ coding sequence in polyA tail-selected EST libraries. Matches with less than $98 \%$ identity were removed. Tomato cDNAs were PCR-tested using EST templates from The Boyce Thompson Institute for Plant Research. The National BioResource Project Tomato (Ministry of Education, Culture, Sports, Science and Technology, Tokyo) provided some of the tomato cDNA clones.

\section{Constructs, plant material and growth conditions.}

All kinases and $P$. syringae effectors were amplified by PCR with gene-specific primers, were cloned into the pENTR (Invitrogen, Green Island, NY, U.S.A.), and were transferred into pAcExAn6 or pDuExAc6 (Fujikawa 2007) using Gateway reactions. Tomato plants used for protoplast extraction were grown in soil in the greenhouse under natural light. Plants were maintained at 21 to $24^{\circ} \mathrm{C}$ and $75 \%$ relative humidity. $N$. benthamiana plants used for VIGS were grown at 22 to $24^{\circ} \mathrm{C}$ for $16 \mathrm{~h}$ light in a growth chamber.

\section{Protoplast extraction, transformation, and split-luciferase assays.}

Briefly, to prepare protoplasts, tomato leaves were cut, placed in protoplast isolation media containing cellulose (Yakult, 
Tokyo) and macerozyme (Yakult) for 12 to $13 \mathrm{~h}$ at $21^{\circ} \mathrm{C}$. The protoplast suspension was passed through a 200-micron sieve, W5 solution was added, followed by centrifugation for $2 \mathrm{~min}$ at $400 \times g$ in an Eppendorf 5810 centrifuge (Eppendorf NA, Hauppauge, NY, U.S.A.). Protoplasts were resuspended in MMG (0.4 M mannitol, $15 \mathrm{mM} \mathrm{MgCl}_{2}, 4 \mathrm{mM}$ MES, pH 5.6) buffer at the final density of $125 \times 10^{4}$ protoplasts per milliliter and were transformed (Kato and Jones 2010). ViviRen (Promega, Madison, WI, U.S.A.) was used as luciferase substrate. Luciferase activity in SLC assays was measured with a Synergy 2 microplate reader (BioTek Instruments, Winooski, VT, U.S.A.). Protoplasts were examined by staining with $0.04 \%$ Evan's blue dye (Sigma, St. Louis) and were observed under a light microscope. For protein detection, protoplasts were pelleted and boiled in sodium dodecyl sulfate (SDS) sample buffer for $5 \mathrm{~min}$. Proteins were analyzed by immunoblotting using an anti-Renilla luciferase Ab (MBL International, Woburn, MA, U.S.A.).

\section{SLC array data analysis for PPI decision.}

For SLC measurements, six data points and four technical replicates were collected per "kinase-effector" set. For data normalization, technical as well as functional controls were defined: background (untransformed protoplasts) and fulllength luciferase (luciferase-transformed protoplasts) measurements were performed independently for each technical replicate and were used for scaling the data. AvrPto-Pto and AvrPto $^{\text {I96A }}$-Pto were used as RIS. The data was represented as vectors in the six-dimensional space, with an $\mathrm{L}^{2}$ norm. Data normalization consisted of subtracting the background measurements and scaling all data according to the intensity of RIS as follows.

$$
\boldsymbol{y}_{N}=\frac{\boldsymbol{y}-\boldsymbol{b}}{\text { mean }\left(\boldsymbol{p}_{C}\right)}, \boldsymbol{y}=\left[y_{1}, \cdots y_{6}\right], \boldsymbol{b}: \text { background, } \boldsymbol{p}_{C} \text { : positive control }
$$

The time series were used to compute distances between fluorescence intensity curves as follows.

$$
d(\boldsymbol{y}, \boldsymbol{x})=\sqrt{\sum_{i=1}^{6}\left(y_{i}-x_{i}\right)^{2}}
$$

The distance of the positive to the negative control was used to test for candidates with significantly higher distances from the negative control level as follows,

$z=\frac{\operatorname{mean}\left(d\left(\boldsymbol{y}_{N}, \boldsymbol{n}_{C}\right)-d\left(\boldsymbol{p}_{C}, \boldsymbol{n}_{C}\right)\right)}{s}, s=\frac{\sigma^{2}}{n}, \sigma^{2}=\operatorname{var}\left(d\left(\boldsymbol{y}_{N}, \boldsymbol{n}_{C}\right)-d\left(\boldsymbol{p}_{C}, \boldsymbol{n}_{C}\right)\right)$

using the testing hypothesis that the candidate's normalized distance from the negative control is not greater than that of positive control, at the $5 \%$ significance level.

\section{Gene silencing and cell death suppression assay.}

VIGS was used in $N$. benthamiana plants as previously described (Ratcliff et al. 2001). In brief, reverse transcription followed by PCR was performed to amplify a $B s k 7$ gene fragment that was cloned into the pCR, a Gateway compatible ENTRY vector (Invitrogen). The fragment was subcloned into the pTRV2 VIGS vector and was transformed into Agrobacterium sp. strain GV2260 for infiltration in N. benthamiana. Real-time PCR using SYBR green (BioRad, Hercules, CA, U.S.A.) was performed to confirm silencing of the $B S K 7$ gene in VIGS plants. Real-time PCR to quantify BSK7 mRNA was performed using the forward primer $5^{\prime}$-gaggttccttctcatgtgttga$3^{\prime}$ and the reverse primer $5^{\prime}$-aaatctcatggatggcagttag- $3^{\prime}$. The primers for the control $F-B O X$ gene were designed as described by Liu and associates (2012). The amount of BSK7 RNA (target) was determined by normalizing the target RNA to the internal standard RNA $(F-B O X)$. The relative expression ratio was calculated by dividing the normalized target RNA values with normalized values of the control plants.

\section{Protein microarray assays.}

For PMA, proteins were expressed and purified as described by Popescu and associates (2007b). The proteins were printed onto PATH slides (Grace Biolabs, Bend, OR, U.S.A.) with a MicroGridPro printer (Biorobotics, Boston). For immunoassays, PMA were blocked in SuperBlock (Pierce, Rockford, IL, U.S.A.) for $1 \mathrm{~h}$ at $4^{\circ} \mathrm{C}$, were incubated with Anti-cMyc Cy3labeled $\mathrm{Ab}$ (Sigma) at a 1:2,500 dilution, were washed in phosphate-buffered saline-T, were dried and scanned in a ScanArrayExpress scanner (Perkin Elmer, Waltham, MA, U.S.A.) at a (photomultiplier tube) strength of $65 \%$, and were analyzed using Genepix Pro 6.0. For interaction assays, PMA were blocked and exposed for $1 \mathrm{~h}$ at $4^{\circ} \mathrm{C}$ to $300 \mu \mathrm{l}$ of interaction buffer (IB: $0.01 \mathrm{M}$ Tris-HCl, pH 6, $0.0002 \mathrm{M}$ ATP, $0.002 \mathrm{M}$ $\mathrm{MgCl}_{2}, 0.002 \mathrm{M} \mathrm{MnCl}_{2}, 0.0001 \mathrm{M} \mathrm{CaCl}_{2}, 0.2 \%$ bovine serum albumin [BSA], $0.0002 \mathrm{M}$ dithiothreitol [DTT]) or IB containing $750 \mathrm{nM}$ AvrPto or $750 \mathrm{nM}$ AvrPto ${ }^{196 \mathrm{~A}}$. Slides were washed and exposed to Anti-V5 Cy3-labeled Ab., 1:1000 (V4014; Sigma) for $1 \mathrm{~h}$ and were processed as above.

\section{In vitro kinase assays.}

Purified kinase $(13 \mu \mathrm{l})$ was mixed with $6 \mu \mathrm{l}$ of kinase buffer (50 mM Tris- $\mathrm{HCl}, \mathrm{pH} 7.5,10 \mathrm{mM} \mathrm{MnCl}_{2}, 10 \mathrm{mM} \mathrm{MgCl}, 1$ $\mathrm{mM} \mathrm{CaCl} 2,1 \%$ BSA, $1 \mathrm{mM}$ DTT, $0.1 \mathrm{mM}$ myelin basic protein, and $1 \mu \mathrm{l}$ of $\gamma \mathrm{P}^{32}$-ATP) and was incubated at $30^{\circ} \mathrm{C}$ with rotation for $30 \mathrm{~min}$. The reaction was stopped by adding SDS loading buffer and boiling for $10 \mathrm{~min}$. The proteins were run on SDS-polyacrylamide electrophoresis gels; the gels were dried in an oven for 30 min and were exposed to Kodak film.

\section{ACKNOWLEDGMENTS}

We are thankful to G. Martin, A. Collmer, P. Boyle, S. Chakravarthy, A. Bombarely, H. Rosli and P. Zuluaga for the helpful discussions and providing materials and reagents used in this research. The authors acknowledge N. Kato for providing the SLC vectors and Plant Genome Research Program (PGRP) Research Experience for Undergraduates students A. Guggisberg, J. Newsome, and K. Morey for contributions to this work. Funding for this research was provided by the National Science Foundation award IOS-1025642 (to S. C. Popescu), IOB-0321453 and IOS-0745545 (to J. W. Stratmann), and National Research Council-Executive Agency for Higher Education, Research, Development and Innovation Funding project number PN-II-PT-PCCA-2011-3.1-1350 (to G. V. Popescu). D. K. Singh developed the protoplast protocols, SLC screens, and contributed to writing; E. K. Brauer performed the PMA work and contributed to writing; N. Fenandez-Pozo, S. Strickler, and L. Mueller carried out the kinome bioinformatics. M. Calvino performed the TOKN cloning; G.V. Popescu carried out the SLC bioinformatics; R. Yalamanchili and J. Stratmann contributed to the protoplast protocol. H. Suzuki, K. Aoki, and D. Shibata contributed reagents for TOKN cloning; S. C. Popescu conceived, designed, supervised the project, and wrote the manuscript.

\section{LITERATURE CITED}

Altschul, S. F., Madden, T. L. Schäffer, A. A., Zhang, J., Zhang, Z., Miller, W., and Lipman, D. J. 1997. Gapped BLAST and PSI-BLAST: A new generation of protein database search programs. Nucleic Acids Res. 25:3389-3402.

Arabidopsis Interactome Mapping Consortium. 2011. Evidence for network evolution in an Arabidopsis interactome map. Science 333:601-607.

Azmi, A.S., Wang, Z.-X., Deng, R.-P., Guo, S.-J., Zhang, J.-B., and Tao, S.-C. 2012. Protein microarray: An ideal platform for systems biology. Pages 113-134 in: Systems Biology, Cancer Research and Drug Discovery. Springer, Dordrecht, The Netherlands.

Bogdanove, A. J., and Martin, G. B. 2000. AvrPto-dependent Pto-interacting proteins and AvrPto-interacting proteins in tomato. Proc. Natl. Acad. Sci. U.S.A. 97:8836-8840. 
Boller, T., and Felix, G. 2009. A renaissance of elicitors: Perception of microbe-associated molecular patterns and danger signals by pattern-recognition receptors. Ann Rev Plant Biol 60:379-406.

Bombarely, A., Menda, N., Tecle, I. Y., Buels, R. M., Strickler, S., FischerYork, T., Pujar, A., Leto, J., Gosselin, J., and Mueller, L. A. 2011. The Sol Genomics Network (solgenomics. net): Growing tomatoes using Perl. Nucleic Acids Res. 39:D1149-D1155.

Chakravarthy, S., Velãsquez, A. C., and Martin, G. B. 2009. Assay for pathogen-associated molecular pattern (PAMP)-triggered immunity (PTI) in plants. J. Vis. Exp. 31. Published online. doi: 10.3791/1442

Day, B., and He, S. Y. 2010. Battling immune kinases in plants. Cell Host Microbe 7:259-261.

Devarenne, T. P., Ekengren, S. K., Pedley, K. F., and Martin, G. B. 2005 Adi3 is a Pdk1-interacting AGC kinase that negatively regulates plant cell death. EMBO (Eur. Mol. Biol. Organ.) J. 25:255-265.

Dou, D., and Zhou, J.-M. 2012. Phytopathogen effectors subverting host immunity: Different foes, similar battleground. Cell Host Microbe 12:484-495.

Edgar, R. C. 2004. MUSCLE: Multiple sequence alignment with high accuracy and high throughput. Nucleic Acids Res. 32:1792-1797.

Faraco, M., Di Sansebastiano, G. P.. Spelt, K., Koes, R. E., and Quattrocchio, F. M. 2011. One protoplast is not the other! Plant Physiol. 156:474-478.

Feng, F., Yang, F.. Rong, W., Wu, X., Zhang, J., Chen, S., He, C., and Zhou, J. M. 2012. A Xanthomonas uridine 5[prime]-monophosphate transferase inhibits plant immune kinases. Nature 485:114-118.

Finn, R. D., Mistry, J., Tate, J., Coggill, P., Heger, A., Pollington, J. E., Gavin, O. L., Gunasekaran, P., Ceric, G., and Forslund, K. 2010. The Pfam protein families database. Nucleic Acids Res. 38:D211-D222.

Finn, R. D., Clements, J., and Eddy, S. R. 2011. HMMER web server: Interactive sequence similarity searching. Nucleic Acids Res. 39:W29W37.

Fujikawa, Y., Kato, N. 2007. Split luciferase complementation assay to study protein-protein interactions in Arabidopsis protoplasts. Plant J. 52:185-195.

Garcia-Hernandez, M., Berardini, T., Chen, G., Crist, D., Doyle, A., Huala, E., Knee, E., Lambrecht, M., Miller, N., and Mueller, L.A. 2002. TAIR: A resource for integrated Arabidopsis data. Func. Integr. Genomics 2:239-253.

Goodstein, D. M., Shu, S., Howson, R., Neupane, R., Hayes, R. D., Fazo, J., Mitros, T., Dirks, W., Hellsten, U., and Putnam, N. 2012. Phytozome: A comparative platform for green plant genomics. Nucleic Acids Res. 40:D1178-D1186.

Horvath, E. 2009. Protoplast isolation from Solanum lycopersicum L. leaf tissue and their response to shortterm $\mathrm{NaCl}$ treatment. Acta Biol. Szeg. 53:83-86

Hou, S., Yang, Y., Wu, D., and Zhang, C. 2011. Plant immunity: Evolutionary insights from PBS1, Pto, and RIN4. Plant Signal. Behav. 6:794799

Jones, J. D. G., and Dangl, J. L. 2006. The plant immune system. Nature 444:323-329

Kato, N., and Jones, J. 2010. The split luciferase complementation assay. Pages 359-376 in: Plant Developmental Biology, Springer, Dordrecht, The Netherlands.

Kaushansky, A., Allen, J. E., Gordus, A., Stiffler, M. A., Karp, E. S., Chang, B. H., and MacBeath, G. 2010. Quantifying protein-protein interactions in high throughput using protein domain microarrays. Nat. Protoc. 5:773-790.

Kim, D., Pertea, G., Trapnell, C., Pimentel, H., Kelley, R., and Salzberg, S L. 2013. TopHat2: Accurate alignment of transcriptomes in the presence of insertions, deletions and gene fusions. Genome Biol. 14:R36.

Lehti-Shiu, M. D., Zou, C., Hanada, K., and Shiu, S.-H. 2009. Evolutionary history and stress regulation of plant receptor-like kinase/pelle genes. Plant Physiol. 150:12-26.

Li, L., Stoeckert, C. J., and Roos, D. S. 2003. OrthoMCL: Identification of ortholog groups for eukaryotic genomes. Genome Res. 13:21782189.

Liu, D., Shi, L., Han, C., Yu, J., Li, D., and Zhang, Y. 2012. Validation of reference genes for gene expression studies in virus-infected Nicotiana benthamiana using quantitative real-time PCR. PLoS One 7:e46451. Published online.

Lu, D., Wu, S., Gao, X., Zhang, Y., Shan, L., and He, P. 2010. A receptorlike cytoplasmic kinase, BIK1, associates with a flagellin receptor complex to initiate plant innate immunity. Proc. Natl. Acad. Sci. U.S.A. 107:496-501.

Martin, F., and Kamoun, S. 2012. Effectors in plant-microbe interactions, Wiley Online Library. doi: 10.1002/9781119949138.fmatter. Published online.

Meng, X., and Zhang, S. 2013. MAPK Cascades in Plant disease resistance signaling. Ann. Rev. Phytopathol. 51:245-266.
Moller, S., Croning, M. D., and Apweiler, R. 2001. Evaluation of methods for the prediction of membrane spanning regions. Bioinformatics 17:646653.

Mueller, L. A., Tanksley, S. D., Giovannoni, J. J., van Eck, J., Stack, S., Choi, D., Kim, B. D., Chen, M., Cheng, Z., Li, C., Ling, H., Xue, Y. Seymour, G., Bishop, G., Bryan, G., Sharma, R., Khurana, J., Tyagi, A., Chattopadhyay, D., Singh, N. K., Stiekema, W., Lindhout, P., Jesse, T., Lankhorst, R. K., Bouzayen, M., Shibata, D., Tabata, S., Granell, A., Botella, M. A., Giuliano, G., Frusciante, L., Causse, M., and Zamir, D. 2005. The Tomato Sequencing Project, the first cornerstone of the International Solanaceae Project (SOL). Compar. Funct. Genom. 6:153158.

Nemoto, K., Seto, T., Takahashi, H., Nozawa, A., Seki, M., Shinozaki, K., Endo, Y., and Sawasaki, T. 2011. Autophosphorylation profiling of Arabidopsis protein kinases using the cell-free system. Phytochemistry 72:1136-1144.

Nguyen, H. P., Yeam, I., Angot, A., and Martin, G. B. 2010. Two virulence determinants of type III effector AvrPto are functionally conserved in diverse Pseudomonas syringae pathovars. New Phytol. 187:969-982.

Nomura, K., DebRoy, S., Lee, Y. H., Pumplin, N., Jones, J., and He, S. Y. 2006. A Bacterial virulence protein suppresses host innate immunity to cause plant disease. Science 313:220-223.

Oh, H. S., and Collmer, A. 2005. Basal resistance against bacteria in Nicotiana benthamiana leaves is accompanied by reduced vascular staining and suppressed by multiple Pseudomonas syringae type III secretion system effector proteins. Plant J. 44:348-359.

Popescu, G., and Popescu, S. 2011. Complexity and modularity of MAPK signaling networks. Pages 355-368in: Handbook of Research on Computational and Systems Biology: Interdisciplinary Applications. D. W. Limin, A. Liu, and Y. L. Hershey, eds. IGI Global, Hershey, PA, U.S.A.

Popescu, S., Snyder, M., and Dinesh-Kumar, S. 2007a. Arabidopsis Protein microarrays for the high-throughput identification of protein-protein interactions. Plant Signal. Behaviour. 2:415-419.

Popescu, S. C., Popescu, G. V., Bachan, S., Zhang, Z., Seay, M., Gerstein, M., Snyder, M., and Dinesh-Kumar, S. P. 2007b. Differential binding of calmodulin-related proteins to their targets revealed through high-density Arabidopsis protein microarrays. Proc. Natl. Acad. Sci. U.S.A. 104:47304735.

Popescu, S., Popescu, G., Bachan, S., Zhang, Z., Gerstein, M., Snyder, M., and Dinesh-Kumar, S. 2009a. MAPK target networks in Arabidopsis thaliana revealed using functional protein microarrays. Genes Dev. 23:80-92.

Popescu, S. C., Popescu, G. V., Snyder, M., and Dinesh-Kumar, S. P. 2009b. Integrated analysis of co-expressed MAP kinase substrates in Arabidopsis thaliana. Plant Signal. Behav. 4:524-527.

Ratcliff, F., Martin-Hernandez, A. M., and Baulcombe, D. C. 2001. Technical Advance: Tobacco rattle virus as a vector for analysis of gene function by silencing. Plant J. 25:237-245.

Reboul, J., Vaglio, P., Rual, J.F., Lamesch, P., Martinez, M., Armstrong, C. M., Li, S., Jacotot, L., Bertin, N., Janky, R., Moore, T., Hudson, J. R., Hartley, J. L., Brasch, M. A., Vandenhaute, J., Boulton, S., Endress, G. A., Jenna, S., Chevet, E., Papasotiropoulos, V., Tolias, P. P., Ptacek, J., Snyder, M., Huang, R., Chance, M. R., Lee, H., Doucette-Stamm, L., Hill, D .E., and Vidal, M. 2003. C. elegans ORFeome version 1.1: Experimental verification of the genome annotation and resource for proteome-scale protein expression. Nat. Genet. 34:35-41.

Rual, J.-F., Hirozane-Kishikawa, T., Hao, T., Bertin, N., Li, S., Dricot, A., Li, N., Rosenberg, J., Lamesch, P., Vidalain, P.-O., Clingingsmith, T. R. Hartley, J. L., Esposito, D., Cheo, D., Moore, T., Simmons, B., Sequerra, R., Bosak, S., Doucette-Stamm, L., Le Peuch, C., Vandenhaute, J., Cusick, M. E., Albala, J. S., Hill, D. E., and Vidal, M. 2004. Human ORFeome Version 1.1: A Platform for Reverse Proteomics. Genome Res. 14:2128-2135.

Rubin, G. M., Hong, L., Brokstein, P., Evans-Holm, M., Frise, E., Stapleton, M., and Harvey, D. A. 2000. A drosophila complementary DNA resource. Science 287:2222-2224.

Sakamoto, T., Deguchi, M., Brustolini, O. J., Santos, A. A., Silva, F. F., and Fontes, E. P. 2012. The tomato RLK superfamily: Phylogeny and functional predictions about the role of the LRRII-RLK subfamily in antiviral defense. BMC Plant Biol. 12:229.

Scofield, S. R., Tobias, C. M., Rathjen, J. P., Chang, J. H., Lavelle, D. T., Michelmore, R. W., and Staskawicz, B. J. 1996. Molecular basis of gene-for-gene specificity in bacterial speck disease of tomato. Science 274:2063-2065

Shan, L., Thara, V. K., Martin, G.B., Zhou, J.-M., and Tang, X. 2000. The Pseudomonas AvrPto protein is differentially recognized by tomato and tobacco and is localized to the plant plasma membrane. Plant Cell 12:2323-2337.

Shan, L., He, P., Li, J., Heese, A., Peck, S. C., Nürnberger, T., Martin, G. B., and Sheen, J. 2008. Bacterial effectors target the common signaling 
partner BAK1 to disrupt multiple MAMP receptor-signaling complexes and impede plant immunity. Cell Host Microbe 4:17-27.

Shi, H., Shen, Q., Qi, Y., Yan, H., Nie, H., Chen, Y., Zhao, T., Katagiri, F., and Tang, D. 2013. BR-SIGNALING KINASE1 physically associates with FLAGELLIN SENSING2 and regulates plant innate immunity in Arabidopsis. Plant Cell 25:1143-1157.

Sreeramulu, S., Mostizky, Y., Sunitha, S., Shani, E., Nahum, H., Salomon, D., Hayun, L. B., Gruetter, C., Rauh, D., Ori, N., and Sessa, G. 2013 BSKs are partially redundant positive regulators of brassinosteroid signaling in Arabidopsis. Plant J. 74:905-19

Tamura, K., Peterson, D., Peterson, N., Stecher, G., Nei, M., and Kumar, S. 2011. MEGA5: Molecular evolutionary genetics analysis using maximum likelihood, evolutionary distance, and maximum parsimony methods. Mol. Biol. Evol. 28:2731-2739.

Tang, W., Deng, Z., Oses-Prieto, J.A., Suzuki, N., Zhu, S., Zhang, X., Burlingame, A. L., and Wang, Z.-Y. 2008. Proteomics studies of brassinosteroid signal transduction using prefractionation and two-dimensional DIGE. Mol Cell Proteomics 7:728-738.

Tang, X., Frederick, R. D., Zhou, J., Halterman, D. A., Jia, Y., and Martin, G. B. 1996. Initiation of plant disease resistance by physical interaction of AvrPto and Pto kinase. Science 274:2060-2063.

The Tomato Genome Consortium. 2012. The tomato genome sequence provides insights into fleshy fruit evolution. Nature 485:635-641.

Trapnell, C., Roberts, A., Goff, L., Pertea, G., Kim, D., Kelley, D. R., Pimentel, H., Salzberg, S. L., Rinn, J. L., and Pachter, L. 2012. Differential gene and transcript expression analysis of RNA-seq experiments with TopHat and Cufflinks. Nat. Protoc. 7:562-578.

Van der Hoeven, R., Ronning, C., Giovannoni, J., Martin, G., and Tanksley, S. 2002. Deductions about the number, organization, and evolution of genes in the tomato genome based on analysis of a large expressed sequence tag collection and selective genomic sequencing. Plant Cell 14:1441-1456.

Walter, M., Chaban, C., Schütze, K., Batistic, O., Weckermann, K., Näke, C., Blazevic, D., Grefen, C., Schumacher, K., and Oecking, C. 2004. Visualization of protein interactions in living plant cells using bimolecular fluorescence complementation. Plant J. 40:428-438.

Wang, H., Chevalier, D., Larue, C., Cho, S. K., and Walker, J. C. 2007. The protein phosphatases and protein kinases of Arabidopsis thaliana. The Arabidopsis Book 5:e0106. Published online.

Wang, Y., Li, J., Hou, S., Wang, X., Li, Y., Ren, D., Chen, S., Tang, X., and Zhou, J.-M. 2010. A Pseudomonas syringae ADP-ribosyltransferase inhibits Arabidopsis mitogen-activated protein kinase kinases. Plant Cell 22:2033-2044.

Xiang, T., Zong, N., Zhang, J., Chen, J., Chen, M., and Zhou, J.-M. 2011. BAK1 is not a target of the Pseudomonas syringae effector AvrPto. Mol. Plant-Microbe Interact. 24:100-107.

Zhang, J., and Zhou, J.-M. 2010. Plant immunity triggered by microbial molecular signatures. Mol. Plant 3:783-793.

Zhang, Z., Wu, Y. Hou, S., Wang, X., Li, Y., Ren, D., Chen, S., Tang, X. and Zhou, J.-M. 2012. Disruption of PAMP-induced MAP kinase cascade by a Pseudomonas syringae effector activates plant immunity mediated by the NB-LRR protein SUMM2. Cell Host Microbe 11:253263.

\section{AUTHOR-RECOMMENDED INTERNET RESOURCES}

Solgenomics website: ftp.solgenomics.net/projects/TOKNv1.0 ea-utils FASTQ processing utilities website: code.google.com/p/ea-utils/wiki/FastqMcf 